\title{
Adverse drug reactions due to immunosuppressants reported in renal transplant recipients in a tertiary care hospital
}

\author{
G. Sirisha ${ }^{1, *}$, Neeraj Sadiq ${ }^{2}$, Y. Venkata Rao ${ }^{3}$ \\ ${ }^{1,2}$ Assistant Professor, ${ }^{3}$ Professor, Dept. of Pharmacology, Kamineni Institute of Medical Sciences, Telangana, India
}

*Corresponding Author: G. Sirisha

Email: dr.sirisha.g@gmail.com

\begin{abstract}
Introduction: Tacrolimus, mycophenolate sodium and prednisolone are used in combination as maintenance treatment in patients who have undergone kidney transplantation. These drugs are used to prevent acute graft rejection.

Aim and Objective: The present study was taken up to assess adverse drug reactions associated with the use of immunosuppressants in patients who have undergone renal transplantation.

Materials and Methods: This was a prospective observational study. 80 patients who had undergone renal transplantation and were attending Post-transplant OPD in department of Nephrology, Nizam's Institute of Medical Sciences, Hyderabad from June 2015 to June 2016 were taken up for the study. These patients were on maintenance treatment with Tacrolimus, Mycophenolate sodium and prednisolone. After taking written informed consent from these patients, relevant information was taken from their investigation reports and discharge summaries.

Results: In the present study, adverse effects were studied in 80 patients (68 males and 12 females) after observing for a period of one year. New onset diabetes after transplantation (40\%) and anemia (20\%) were most common adverse effects. Infections occurred in $31.25 \%$ patients, out of which CMV infection and UTI caused by E.coli were most common. Nephrotoxicity and electrolyte disturbances such as hyperkalemia were also frequently reported.

Conclusion: Results of this study show that immunosuppressants used in post kidney transplant patients may cause serious and frequent adverse effects. Monitoring and regular follow up of these patients is therefore necessary.
\end{abstract}

Keywords: Immunosuppressants, Kidney transplant, Adverse effects, Frequency, Infections.

\section{Introduction}

Immunosuppressant drugs are used in solid organ transplantation to prevent rejection of transplanted organs. These drugs have narrow therapeutic index and wide interindividual variability in dose requirement. Overdosing of immunosuppressants increases the risk of infection and drug specific complications such as new onset diabetes after transplantation such as that caused by tacrolimus. ${ }^{1,2}$ Episodes of graft rejection may occur, if the dose is not sufficient.

The basic immunosuppressive protocols contain multiple drugs being administered simultaneously. Therapy typically involves calcineurin inhibitor such as tacrolimus, glucocorticoids and mycophenolate mofetil sodium, each directed at a discrete site in T cell activation. ${ }^{3}$

There is inadequate information regarding the adverse effects observed during the use of immunosuppressants, especially among the South Indian renal transplant population. So, the present study was conducted to find the incidence of adverse effects experienced by the patients in the post renal transplant period.

\section{Materials and Methods}

The study was a prospective observational study conducted on 80 patients, who have undergone renal transplantation and were on maintenance immunosuppressive regimen containing tacrolimus (2-5 $\mathrm{mg} /$ day), mycophenolate mofetil sodium $(720 \mathrm{mg}$ twice daily) and prednisone (5-7.5mg/day).

The study was conducted on patients attending outpatient transplant clinic in Department of Nephrology in Nizam's Institute of Medical Sciences, Hyderabad. The duration of the study was 1 year (June 2015- June 2016). The study started after taking permission from NIMS Institutional Ethics Committee TAC/PG/01, version 1 dated 16-6-2015). After taking written informed consent from these patients, relevant information was taken from their investigation reports and discharge summaries. Adverse drug reactions were then classified according to their severity. The patients were followed for a period of one year.

\section{Results}

In the present study, 80 renal transplant patients who were on immunosuppressants were included. Among the patients enrolled, 68 were male patients and 12 were female patients. The demographic details of the patients were recorded and tabulated. The age of the patients was in the range of 18 to 63 years. The weight of the patients was in the range of 40 to $78 \mathrm{~kg}$.

Table 1: Demographic details of renal transplant patients

\begin{tabular}{|c|c|c|}
\hline Gender & Males $(\mathbf{n}=\mathbf{6 8})$ & Females $(\mathbf{n = 1 2})$ \\
\hline Age in years $($ Mean \pm SEM $)$ & $27 \pm 9.9$ & $30 \pm 0.42$ \\
\hline BMI in $\mathrm{kg} / \mathrm{m}^{2}($ Mean \pm SEM $)$ & $20.1 \pm 3.5$ & $19.2 \pm 0.8$ \\
\hline
\end{tabular}


The patients had normal BMI (20.92 \pm 3.54$)$ (Table 1).

Table 2: Age wise distribution of patients having undergone kidney transplantation

\begin{tabular}{|c|c|}
\hline \multicolumn{1}{|c|}{ Age } & No of patients \\
\hline $10-20$ & 2 \\
\hline $20-30$ & 46 \\
\hline $30-40$ & 20 \\
\hline $40-50$ & 9 \\
\hline $50-60$ & 2 \\
\hline $60-70$ & 1 \\
\hline
\end{tabular}

The majority of patients were in the age group of 20-30 years $(n=46)$, followed by age groups 30-40 $(n=20)$ and 40 $50(n=9)$ respectively (Table 2$)$.

Chronic interstitial nephritis, malignant hypertension, IgA nephropathy and crescentic glomerulonephritis are common kidney diseases that lead to end stage renal disease resulting in transplantation.

Table 3: Adverse drug reactions with immunosuppressants

\begin{tabular}{|l|c|c|}
\hline Adverse drug reaction & $\begin{array}{c}\text { Number of } \\
\text { patients }\end{array}$ & $\begin{array}{c}\text { Frequency } \\
(\%)\end{array}$ \\
\hline $\begin{array}{l}\text { New onset diabetes after } \\
\text { transplantation } \\
\text { (NODAT) }\end{array}$ & 32 & 40 \\
\hline Anaemia & 16 & 20 \\
\hline Leukopenia & 2 & 2.5 \\
\hline Nephrotoxicity & 8 & 10 \\
\hline Electrolyte disturbances & 7 & 8.75 \\
\hline Infections & 25 & 31.25 \\
\hline Raised serum bilirubin & 3 & 3.75 \\
\hline $\begin{array}{l}\text { Raised serum } \\
\text { cholesterol levels }\end{array}$ & 2 & 2.5 \\
\hline
\end{tabular}

New onset diabetes after transplantation (NODAT) was commonly seen in as many as $40 \%$ of the patients. Electrolyte disturbances and nephrotoxicity with elevation of serum creatinine were also observed in the patients. Anemia was seen in $20 \%$ of the post transplant patients (Table 3).

Infections were common (31.25\% of patients), as the patients were on immunosuppressants. The various infections observed in the patients were tabulated in table 4.

Table 4: Infections with the use of immunosuppressants

\begin{tabular}{|l|c|c|}
\hline \multicolumn{1}{|c|}{ Infection } & $\begin{array}{c}\text { Number of } \\
\text { patients }\end{array}$ & $\begin{array}{c}\text { Frequency } \\
(\boldsymbol{\%})\end{array}$ \\
\hline CMV & 15 & 18.75 \\
\hline UTI $($ E.coli) & 5 & 6.25 \\
\hline Acinetobacter & 2 & 2.5 \\
\hline Enterobacter cloacae & 1 & 1.25 \\
\hline Hepatitis B & 1 & 1.25 \\
\hline Staphylococcus sepsis & 1 & 1.25 \\
\hline
\end{tabular}

Infection with cytomegalovirus was commonly observed in 15 patients. UTI infections due to E.coli were seen in 5 patients (Table 4). Acinetobacter infection was noted in 2 patients. E.cloacae, hepatitis B and Staphlylococcus sepsis was reported in 1 patient each. All patients received co- trimoxazole prophylaxis.

Some patients in the study had electrolyte disturbances, which were as follows (Table 5)

Table 5: Electrolyte disturbances with immunosuppressants

\begin{tabular}{|l|c|c|}
\hline $\begin{array}{c}\text { Electrolyte } \\
\text { disturbance }\end{array}$ & $\begin{array}{c}\text { Number of } \\
\text { patients }\end{array}$ & $\begin{array}{c}\text { Frequency } \\
(\boldsymbol{\%})\end{array}$ \\
\hline Hyperkalaemia & 4 & 5 \\
\hline Hypokalaemia & 2 & 2.5 \\
\hline Hyponatremia & 1 & 1.25 \\
\hline
\end{tabular}

The adverse effects noted were categorized into groups based on their severity (Table 6)

Table 6: Categorization of adverse effects based on severity

\begin{tabular}{|l|c|c|}
\hline Severity & $\begin{array}{c}\text { Number of } \\
\text { patients }\end{array}$ & Frequency (\%) \\
\hline Mild & 47 & 58.75 \\
\hline Moderate & 28 & 35 \\
\hline Severe & 5 & 6.25 \\
\hline
\end{tabular}

\section{Discussion}

Adverse effects following immunosuppression in renal transplant recipients were studied in a total of 80 patients. 5 patients had episodes of graft rejection. 28 patients had moderate adverse effects requiring medical intervention. Other patients had mild adverse effects. ${ }^{4}$ No serious adverse effects or deaths were reported among these patients during the period of study.

New onset diabetes mellitus after transplantation (NODAT) is a serious and common complication following solid organ transplantation. NODAT has been reported to occur in $2 \%$ to $53 \%$ of all solid organ transplants ${ }^{5}$. The development of NODAT has also been shown to be associated with an adverse impact on patient survival and an increased risk of graft rejection and graft loss. ${ }^{6}$

Immunosuppressive medications contribute to early and late post transplantation anemia. The mechanism is hypothesized to involve decreased proliferation of erythroid precursors or interference in iron homeostasis. ${ }^{7}$ Winkelmayer et al reported an association between tacrolimus use and anemia. The vasoconstrictive property of tacrolimus that impair renal function and, subsequently, erythropoetin production may be the factors responsible for anemia.

There is increased risk of infection because of decreased immune function. Indeed, infectious complications are reported to be the most common causes of morbidity and mortality after transplantation. ${ }^{8}$ Improved 
immunosuppressive regimens, though have reduced the incidence of allograft rejection, have increased the incidence of susceptibility to opportunistic infections. Other factors that increase risk of infections in post transplant period include malnutrition, co-morbidities associated with end stage renal or liver disease and alterations of the mucocutaneous barriers following the transplant procedure.

In the present study, 5 patients had episodes of graft rejection. Vascular dysfunction results from an increase in vasoconstrictor factors such ad endothelin and thromboxane as well as activation of the renin-angiotensin system (RAS). Reduction of vasodilator factors like prostacyclin, prostaglandin E2, and nitric oxide results in calcineurin inhibitor (CNI) induced nephrotoxicity. ${ }^{9}$ In addition, free radical formation is implicated in acute CNI nephrotoxicity, as well as sympathetic nerve activation in kidneys. Finally, reversible tubular dysfunction is also a feature of acute CNI nephrotoxicity.

In the present study, $8.75 \%$ of patients had electrolyte disturbances. Electrolyte disturbances can be explained by reduced expression of the $\mathrm{Na}^{+}-\mathrm{K}^{+}-2 \mathrm{Cl}^{-}$-cotransporter (NKCC2) at the apical membrane of tubular epithelial cells. $^{10}$

\section{Conclusion}

The common complications of immunosuppressants used in renal transplantation were studied in South Indian patients attending tertiary care hospital. The present study indicates that incidence of NODAT is high, so identification and prevention of risk factors for NODAT as well as treatment of hyperglycemia is important. Anemia, infections, nephrotoxicity and electrolyte disturbances were also commonly seen in these patients. Monitoring the patients for these adverse effects and instituting early treatment is essential to prevent further complications.

\section{References}

1. Guruprasad P, Kishore K, Mahajan S, Aggarwal S. Active surveillance for adverse events among patients who underwent renal transplantation: A prospective observational study. Perspect Clin Res. 2017;8:118-123.

2. Pasha AA. Side Effects of Transplant Immunosuppressive Therapy in Post Renal.

3. Transplant Recipients, Mazandaran, Northern Iran. Zahedan J Res Med Sci. 2017;19(4):6560.

4. Zhang R. Modern Immunosuppressive Therapy in Kidney Transplantation. Open J Organ Transplant Surg. 2013;3:2231.

5. Sharma Love, Kanodia Lalit, Singh Lokesh, Yadav Virendra, Thomas Binny, Hail Moza. A study of adverse events associated with the use of Immunosuppressive agents in kidney transplanted Patients. Int J Drug Dev \& Res. 2012;4(3):283291.

6. PTT Pham. New onset diabetes after transplantation (NODAT): an overview Diabetes, Metabolic Syndrome and Obesity: Targets and Therapy. 2011:(4):175-186.

7. Sneha Palepu, G V Ramesh Prasad. New-onset diabetes mellitus after kidney transplantation: Current status and future directions. World J Diabetes. 2015;6(3):445-455.

8. Winkelmayer WC, Yabu JM. Post transplantation Anemia: Mechanisms and Management. CJASN. 2011(6):71794-1801.
9. Karutu S, Blumberg EA. Common Infections in Kidney Transplant Recipients. Clin J Am Soc Nephrol. 2012(7):20582070.

10. Kuypers DRJ, Sarwal M. Calcineurin Inhibitor Nephrotoxicity. Clin J Am Soc Nephrol. 2009(4):481-508.

11. Lee $\mathrm{CH}, \mathrm{Kim}$ GO. Electrolyte and Acid-Base Disturbances Induced by Clacineurin Inhibitors. Electrolyte Blood Pressure. 2007(5):126-130.

How to cite this article: Sirisha G, Sadiq N, Rao Y. V. Adverse drug reactions due to immunosuppressants reported in renal transplant recipients in a tertiary care hospital. Indian $\mathrm{J}$ Pharm Pharmacol. 2018;5(4):176-178. 